\title{
Research on the Reform of Education under the Background of Big Data
}

\author{
Jiang Pan \\ Information Engineering Institute, Wuhan Business University, Wuhan, China \\ 44257597@qq.com
}

Keywords: Fragmented learning, Educational big data, Reform.

\begin{abstract}
With the innovation of Internet technology, big data begins to spread to various industries and fields, affects people's knowledge system and way of life. In the era of big data, how to stimulate and use the undiscovered value of data and achieve innovation in education, economics, transportation, medical and other fields is exploring by national education sector and business community in recent years. This paper introduces the concept of big data, the education in the background of big data, and the broad market in the field of education, analysis the change of education brought about by big data, looks forward new opportunities for education in the era of big data.
\end{abstract}

\section{Introduction}

In recent years, with the rapid development and large-scale popularization of sensing technology, social networks and mobile devices, more and more people contact in the network with others, which led directly to the exploding growth of Internet data, human beings had entered the era of big data unknowingly. In 2012 the United Nations released Big Data for Development: Challenges \& Opportunities, which pointed out that the era of big data has come, and the emergence of big data will have a profound impact on all areas of society (1). Therefore, lots of countries invest a lot of manpower and resources to support the research and application of big data. The United States, as a technology powerful country, launched the Big Data Research and Development Initiative in March 2012 , formally raised the research of big data to the national strategic level and invested $\$ 200$ million for the project (2). In the field of education, Yale University, Harvard University, Stanford University and other world-renowned universities have also launched related researches of big data; In addition, the world's leading academic journals Nature and Science respectively in 2008 and 2011 launched special issue related big data, thus, big data has been attached great importance by the education, academia, industry and government agencies of many countries.

\section{The concept of big data}

When people mention big data many people can only descript the scale of big data, such as, dozens of PB data a day dealed with by Baidu; more than 300TB daily log data generated by Facebook; the amount of data does not distinguish between big data and traditional mass data.

In the special issue published in the journal Science in 2008, big data is defined as "represents the progress of human cognitive processes, the size of the data set is unable to obtain, manage, deal with by the current technology, methods and theory " (3). The influential company Gartner also gives a big data definition (4), which is high-volume, high-generating rates, widely variety information value, and new forms of processing are needed to ensure that judgments are made, and insight is found and processing is optimized. the $4 \mathrm{~V}$ definition of big data is that volume, variety, velocity, value, The definition of the fourth $\mathrm{V}$ is not uniform, International Data Corporation believes that the big data should have value (5), the value of big data often show sparse characteristics; And IBM believes that large data must be veracity (6), which is conducive to the establishment of trust mechanism and the leader's decision-making. The definition of big data made by Baidu Encyclopedia is huge amounts of material, meaning that the huge scale of data involved in material can not be captured, managed, processed and sorted through the current mainstream software to help make business decision in a 
reasonable time. Big data scientist Rauser mentions a simple definition that big data is the huge amount of data beyond the computer processing power.

\section{The education in the background of big data}

\subsection{The status of educational big data at home and abroad}

In 2012,United Nations issued a white paper named Big Data for Development: Challenges and Opportunities, which pointed out that the emergence of large data will have a profound impact on various areas of society; In 2013, the education sector of China set off a reform to promote education; In March 2014, Education Offices in Chinese released the paper named Education Information Work in 2014, which required the integration and integration of data resources such as dynamic monitoring, decision-making and educational forecasting to provide timely and accurately data support for educational decision-making. September 5, 2015, the State Council officially released the paper named to promote large data development action plan, which planned 10 big data projects, and in the project named public service big data clearly puts forward to build educational and cultural data; September 19, 2015, China Education Big Data Research Institute was established in Qufu Normal University; In December 2016 , the first big data education forum was held in Beijing University to explore data science and big data personnel training and system construction. The research of big data field of education in China is in full swing to start driven by many policy and practice.

November 2016, Big Data and the Future of Education - International Seminar on Big Data-Driven Education Reform and the First Chinese Education Big Data Development Forum held in Qufu Normal University. The forum is organized by the Qufu Normal University, invited the experts and scholars more than 300 people from various academic institutions and universities across the country, and the United Kingdom, the United States, Australia, Austria, Taiwan. Big data has laid the foundation for the improvement of educational research, decision-making and management, and the excavation and analysis of big data in education is the new direction of understanding and exploring the law of education.

\subsection{Improvement of teaching mode in the background of big data}

With the rapid development of Internet information technology, big data has become the focus of attention, education as an important application area of big data will undergo a revolutionary change. In the field of education the use of big data can improve student achievement, and provide students with personalized service. Through the analysis of big data, we can find the important information neglected in the conventional research, reform the teaching mode of teachers, improve the students' learning effect and optimize the way of education policy. At present, online education and large-scale open-ended web courses are typical applications of big data in education.

The National Center for Educational Statistics and other government agencies have begun to collect student learning behavior test scores, career planning and other important information in the educational institutions, and analysis them with big data methods. Through using big data the US Department of Education has created a learning analysis system, established the joint framework of data mining, data modeling and typical case to provide more information to educators and help them answer the real problem that how to learn.

In order to comply with this trend, the US Federal Ministry of Education carried out an big data plan in 2012, which spent $\$ 200$ million to promote the use of big data in improving education. April 10, 2014, the US Federal Ministry of Education Technical Office released part of the program summary data and cases, and invested $\$ 25$ million from budget for education data mining and learning analysis

\subsection{Large market prospects for education}

Some companies in the United States have succeeded in achieving commercialization of big data processing in education. For example, IBM, the world's largest IT maker, works with the Mopai County Public School District in Alabama to improve student achievement through student data detection and behavioral interventions. With IBM's technical support, the company has established a cross-school learning database that collects records of more than 1 million student and data of more 
than 7 million course records. Software analysis results not only show student achievement, attendance, dropout rate, enrollment rate, but also allow users to detect the warning signal predictively leading student to dropping out of school and declining academic performance, also allow users to find those who lead to unnecessary consumption of specific courses, reveal what kind of resources and intervention is the most successful; By monitoring the students' progress of reading electronic materials, network communication, electronic version of the submission of work, online testing, teachers can promptly diagnose the problem of each student in order to make suggestions timely.

In the future education, the trend of high education will be personalized learning. In the new era of individualization of education, course materials will adapt to meet the unique needs of each student's learning, e-education, its salient features are online education, active education. The analysis of big data can be applied to data mining in education, at present, educational institutions have accumulated a large number of unstructured and structured data, so that researchers have more new opportunities to explore the student learning environment. By monitoring those information can format a large database of education, sum up the law of education further, help teachers understand students, adjust the education program and master the whole learning process of students to improve students' learning outcomes.

In short, applications of big data education can provide students with a tailor-made personalized learning style, an early warning system of educational problems and a flexible and adjust education system provide a new means for teachers to understand the learning ways of students.

\section{Educational reform in the background of big data}

The arrival of big data age, lots of areas are impacted, which leads peoples' attention from the macro to the micro individual. For the field of education, change occurs quietly, such as, the application of big data make it possible to study individual learning, train especially, and promote the development of human beings more than ever.

First, making a multivariate assessment of student development. There are two ways to get good grades, one is to rely on excellent logical thinking ability, the other one is a kind of rely on memory. Relying on memory can achieve good results, but can not develop students' advanced thinking ability, Relying on memory to achieve good results can cover up the shortcomings of students' learning process, big data can distinguish between these two differences. Identifying and distinguishing this difference will help educators to provide targeted help to the students timely and discover issues that are not reflected in the results.

With the increasing demand for talented people, the society calls for an educational idea that can cultivate more individuality and innovative talents and adapt them to the future information and the transformation and development of the knowledge. In this context, individualized education has become a hot topic at home and abroad, The UK government proposes in the Vision 2020 that every student should be protected from race, gender and family background to make education fair; [24] in 2010 Chinese government has promulgated the paper named National Medium and Long Term Education Reform and Development Plan (2010-2020), which puts forward that Concerning about the different characteristics and personality differences of students, developing each student's potential advantages. (7) To build a personalized education environment for students according to the students' practical learning situation can carry out targeted learning guidance, make students into the learning process, which requires to assess the preparation, interest, talent, and learning style of students. Due to the lack of means to get and analysis information, that is, the lack of understanding of the characteristics of the educated and personality differences, we cannot support a learning environment which can provide for the promotion of academic performance and emotional communication. The introduction of Education Institute of Carnegie Mellon University is that we have to admit that we know too little about students. (8)The problem that in the traditional data age have troubled educators have technology and ideas to solve in the big data age. 
Second, achieving to assess the learning process of students. Education in the big data age can monitor the student learning process, find the normal learning style of students, through the analysis of changes in data educators can observe the effect of transformation of class to reflect the way they can improve; Through the analysis of students' learning behavior record can capture students' micro-behavior in the classroom, achieve the combination of big data and the class, understand the interest of students , and promote the reflection of teaching effectiveness.

As one of the important subjects of modern education research, educational evaluation is also an important reference index to evaluate the students' learning effect. The thinking mode of the big data age provides a new way for it which is forming the concept of developmental education evaluation, emphasizing on the purpose of the development of the education evaluation's object, starting from the needs of students, paying attention to learning process, learning experience and communicate between teacher and student. This requires timely and effectively grasp the learning process of students, the achievements and the emotions, attitudes, strategies and the development of other aspects. It is a major problem in the development of educational evaluation that how to obtain the information generated in the class, school assignment, the interaction between teacher and student or between student and student. All kinds of computers and mobile terminals all the time record about people thinking, decision-making and behavior by the Internet and the mobile Internet. Big data has the ability to focus on each object of the educational evaluation in learning activities, such as the teaching content stimulating students to put question, the time to stay in the teacher and so on. It is particularly important that these data are quietly carried out with the support of specific observing techniques and equipment, and the behavior that students do not want to appear in an experiment will get a complete presentation. Students' daily learning and life are not affected, so the data are more real.

Third, accumulate monitoring the learning track of students. Parents can use the mobile phone to obtain the notice of school, record the students' family study status, obtain valuable research report through the regional data statistics, master the students extracurricular learning trajectory, strengthen the students learning atmosphere construction.

In the era of big data, students leave a series of digital debris in the process of learning and living, such as course selection, online learning, interaction and feedback, online social behavior, the use of campus cards, etc., which are integrated and analyzed, the learner's behavior mode will be revealed. Whether it is Arizona State University provides by the personalized learning experience through mining Facebook page data, or East China Normal University develops family financial difficulties student early warning system through the campus card records, are big data thinking applies to education and practice. Big Data Age Personalized ways of thinking can help educational researchers re-examine the needs of students, through the analysis of high-tech and meticulous to find appropriate courses, classrooms, teachers, campus to achieve true sense of education.

Fourth, the learning way affected by educational big data promotes fragmented learning. Chris Anderson, editor-in-chief of the United States magazine, wrote the concept of the Long Tail in the paper of Long Tail in October 2004, whose basic view is that even if the product is very low demand(the tail of the product's normal curve), as long as the market has to sell, there will be someone to buy. The common market share of these products and products with low sales can be comparable to or even larger than the mainstream products (the head of the product's normal curve). (9) The relationship between the degree of knowledge fragmentation and the degree of learners' attention in the field of education is similar to the long tail of the product. As shown in Fig. 1, learners in the traditional teaching model often require less knowledge of fragmentation and lack of attention, resulting in the sense of getting fragmented knowledge is not strong. However, the total amount of knowledge that is required less and is easily overlooked is similar to the total amount of systematic knowledge, even more in depth and breadth than the total amount of systematic knowledge. 


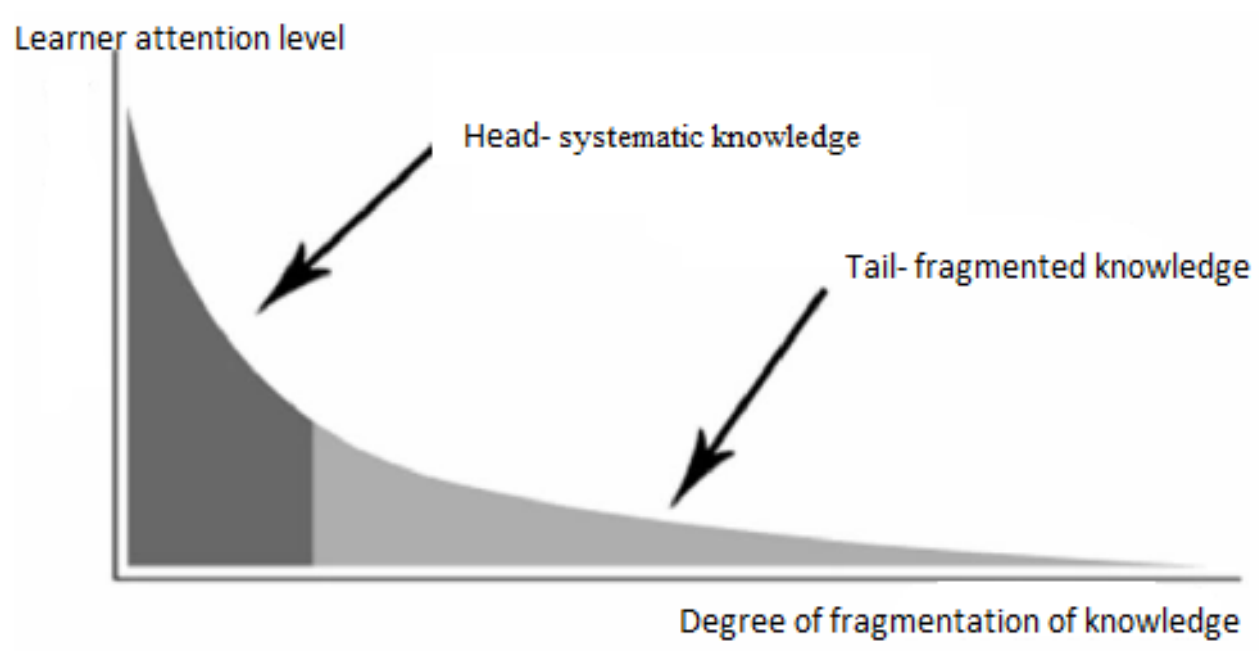

Fig. 1. The long tail theory in education

Most of learners in the traditional teaching model can only learn the important knowledge that educators think in the class, and the probability to expand knowledge independently is very small. If we use the normal distribution curve in Figure 1 to describe this phenomenon, that most of learners under the guidance of teachers, can only focus on the systematic knowledge in the curve head, and the fragmentation of knowledge in the curve tail which need more fragmented energy and time to focus on is often ignored, which is undoubtedly a big loss for learners who need their own growth and the growth of social knowledge. At the same time, fragmentation is also a diversification, learners systematically accept the contents of the program plan or their own professional knowledge, there are more knowledge outside of their cognitive field worth learning, development and mature of educational big data makes this diversified form of learning is possible.

In short, educators and educational institutions in the era of big data should focus on cultivating learners' fragmented learning thinking and create a learning environment for learners supported by big data. Learner in the era of big data should have the ability to learn from fragmented learning and the ability to use educational data to support learning, use fragmented time to get fragmentation knowledge in the end of the curve by mobile learning devices such as smart phones, tablets, PDA, and so on, further obtain in accordance with or even greater than systematically professional knowledge.

\section{Opportunities and challenges of education in the era of big data}

Big data plays a vital role in the change of education and provides a reference for the change of education. It is the optimal solution to realize the individual learning by using the large data analysis to discover the model in the learning process and to intervene the learning process of the students based on it. Learning model big data provides a path and direction for educational analysis, which can solve the problem of lack of comprehensive and pertinent analysis of current educational data analysis and strengthen its combination with teaching. Of course, in the information technology and educational big data for the field of education provide opportunities, but also bring risks and challenges.

Firstly, the application of educational big data makes the information more transparent and enables students and teachers to have more to know. But at the same time, privacy has become a problem. What kind of procedure and supervision is necessary for the access and authority of educational data is a new problem facing the information age, which is closely related to the classification and collection process of data.

In the era of big data, the value of data is getting larger and larger, face of massive data collection, storage, management, analysis and sharing, information security has become the most important issue, prevent data from being stolen or tampered with. The massive data of big data, usually using cloud storage, scattered data management, were unable to control the user location data processing, it is difficult to distinguish between legal and illegal users, and users can easily lead to illegal intrusion, 
theft or tampering with the important data information. How to ensure the security of big data and the reliability of the analysis results is a new topic in the field of information security, prevent personal information leakage, big data includes a large number of personal privacy, as well as detailed records of all kinds of behavior. How to dig the wisdom part not only bring benefits to mankind, and fully protect the personal privacy is not abused, find the balance of personal information protection and open in the use of big data is a huge problem of big data.

Secondly, in the education of big data culture, the analysis of patterns can support the whole field of vision and make the education process integrated and planned. However, there are difficulties and uncertainties in the analysis and interpretation of patterns. This requires not only the support of analytical techniques, but also the understanding of the teaching process, student characteristics, and cultural patterns of educators.

Thirdly, in the application of educational big data, the automatic process data can make the evaluation fairer, and replace the monotonous grade evaluation with the formation evaluation of multidimensional classification. Online data alone, however, does not reflect the whole picture of learning life. How to expand the dimensions and methods of data collection and combine online data with traditional learning process is a big challenge for big data applications.

Finally, the application of big data research promotes the data-driven teaching decision-making and management process innovation. In the teaching practice, the data-driven teaching decision needs the teacher's mastery of the information technology, and the deep understanding of the learning analysis and the information teaching model. However, the application ability of information technology and the concept of information teaching of teachers should be improved, and the teachers are not ready, which will affect the practical value of big data technology.

\section{Conclusion}

We believe that the education of big data is the means of education, not the subject of education, not the purpose of education, and its function is to do its best to provide accurate service for the teaching objects. We will not be able to borrow big data as education technology boost knowledge utilitarian tool, will not be able to borrow big data as a new technology education gimmick to attract the attention of others. If we want to truly achieve educational big data driven education reform, we should base ourselves on the local, and strengthen the collection and analysis of the big data of education. This paper introduces that the education and big data era of big data make broad market application in the field of education, analyzes the changes brought about by the era of big data, look forward to the new development opportunities brought about by the big data for education. The purpose of using educational data is to educate people, and big data is properly recognized and applied in the field of education, so as to better serve the education of people.

\section{Acknowledgments}

Supported by Science and Technology Research Project of Hubei Provincial Department of Education (B2017278). Research on intelligent navigation system based on Data Mining.

\section{References}

[1] Big Data for Development: Challenges \& Opportunities [DB/OL],2012, http://www. unglobalpulse.org/sites/default/files/BigData for Development-UNGlobal Pulse June2012.pdf.

[2] Big Data Research and Development Initiative [DB/OL], 2012.http: //www. White house .gov/sites/default/files/microsites/ostp/big_data_press_release_final_2.pdf.

[3] Graham-Rowe D, Goldston D, Doctorw C,et al. Big data:science in the petabyte era [J], Nature, 2008, 455( 7209), ,8-9. 
[4] JI Chang-qing, LI Yu, QIU Wen-ming, et al. Big data processing in cloud computing environments [C] / / Proc of the 12th International Symposiumon Pervasive Systems, Algorithms and Networks. 2012, 17-23.

[5] Barwick H, The "four Vs" of big data [EB/OL] .2012. http://www. computerworld. com/au/article/396198/iii3_four_vs_big_data/.

[6] IBM. What is big data? [EB /OL ] . [2012-10-02] . http: //www-01. ibm. com / software / data / bigdata / what-is-big-data. html.

[7] Viktor Mayer-Schonberger. Big Data and the Future of Education [R].QuFu:International Seminar on Big Data-Driven Education Reform and the First Chinese Education Big Data Development Forum,2016.

[8] National Medium and Long Term Education Reform and Development Plan (2010-2020) [EB/OL].http://www.gov.cn/jrzg/2010-07/29/content_1667143.htm.

[9] Opportunities and challenges for education brought about by big data [EB/OL]. http:// www. age06.com/Age06.Web/Detail.aspx?InfoGuid=ec46cae7-e39b-4da1-ae0f-980fe4d68929.

[10]Anderson C. The Long Tail [J]. Long Tail - Business Book Summaries, 2004, 11 (1) :1-20.

[11] Yihua Chen. The application of PISA2012 data: on the training mode of the future career ability of Chinese teenagers[R]. QuFu:International Seminar on Big Data-Driven Education Reform and the First Chinese Education Big Data Development Forum, 2016.

[12]Mao Li. The educational vision of Britain in the 2020 [J]. social work, 2008.

[13]Patricia Schwerdtle, James Bonnamy, Big Data in Nurse Education, Nurse Education Today,2017

[14] Tingting Tong, Haizheng Li,Demand for MOOC - an application of Big Data, China Economic Review.2017

[15] Yeon Hee Kim, Jin-Ho Ahn, A Study on the Application of Big Data to the Korean College Education System, Procedia Computer Science,2016 